\title{
X-ray Study of Phason Strains in an AlCuFeMn Decagonal Phase
}

\author{
Wang Yang ${ }^{1}$, Tomoyo Ando $^{2, *}$ and Kazuki Yamamoto ${ }^{2}$ \\ ${ }^{1}$ College of Science, Zhejiang Sci-Tech University, Hangzhou 310018, China \\ ${ }^{2}$ Department of Physics, Nara Women's University, Nara 630-8506, Japan
}

The peak profiles, shifts, and splittings of Bragg reflections, which are inherent in the phason strain of a quasicrystal, are carefully examined using a precise powder X-ray diffraction method for a wide range of different AlCuFeMn alloys and annealing temperatures. Based on the peak-shift dependences of the Bragg reflections on the phason momentum $Q_{\perp}$, two kinds of new approximant phases are identified. These phases have linear phason strains $\theta_{1}=\tau^{-6}$ and $\theta_{2}=\tau^{-5}$, and $\theta_{1}=\tau^{-10}$ and $\theta_{2}=\tau^{-5}$, corresponding to lattice spacings $a=3.814 \mathrm{~nm}$ and $b=2.005 \mathrm{~nm}$, and $a=9.985 \mathrm{~nm}$ and $b=2.005 \mathrm{~nm}$ in the quasiperiodic plane of the orthorhombic system, respectively. [doi:10.2320/matertrans.M2016446]

(Received December 26, 2016; Accepted March 13, 2017; Published April 21, 2017)

Keywords: quasicrystal, linear phason strain, decagonal phase, approximant, AlCuFeMn

\section{Introduction}

The broadening and shifting of Bragg reflection peaks by characteristic strains are of great relevance to quasicrystal stability and to the transformation mechanism from a quasicrystal to an approximant crystal phase. A suitable linear phason strain can transform a decagonal quasicrystal to a one-dimensional quasicrystal or to an approximant crystal, and cause peak shifts that increase monotonically with the phason momentum $Q_{\perp}{ }^{1)}$. We have studied the broadening and shifting of Bragg reflection peaks for decagonal phase of $\left.\mathrm{Al}-\mathrm{Ni}-\mathrm{Co}^{2,3}\right)$ and $\mathrm{Al}-\mathrm{Pd}-\mathrm{Mn}^{4)}$ by the high-resolution single-crystal X-ray diffraction.

In an AlCuFeMn alloy system, Tasi et al. ${ }^{5)}$ discovered a one-dimensional quasicrystal composed of $\mathrm{Al}_{65} \mathrm{Cu}_{20} \mathrm{Fe}_{10} \mathrm{Mn}_{5}$ with a characteristic periodicity of $1.84 \mathrm{~nm}$. Furthermore, Yang et $a l .{ }^{6)}$ discovered two new one-dimensional quasicrystals, also composed of $\mathrm{Al}_{65} \mathrm{Cu}_{20} \mathrm{Fe}_{10} \mathrm{Mn}_{5}$, with characteristic periodicities 2.99 and $4.14 \mathrm{~nm}$. And they discussed the relation between linear phason strains and the peak shifts observed in these phases, and showed that these three phases, with lattice constants $1.84,2.99$, and $4.14 \mathrm{~nm}$, have linear phason strains of $\tau^{-6},-\tau^{-8}$, and $-\tau^{-6}$, respectively.

Until now, neither the ideal decagonal phase nor any related approximant phase has been found in an $\mathrm{AlCuFeMn}$ alloy system. The aim of the present work is therefore to search for this ideal decagonal phase or for some new decagonal phase showing an appropriate linear phason strain and to discuss the phason strains and phase transformations, in a wide range of $\mathrm{AlCuFeMn}$ alloys with different compositions to $\mathrm{Al}_{65} \mathrm{Cu}_{20} \mathrm{Fe}_{10} \mathrm{Mn}_{5}$.

\section{Experiment}

$\mathrm{AlCuFeMn}$ alloy ingots, formed in the composition area with manganese much more preferentially than the one-dimensional quasicrystal $\mathrm{Al}_{65} \mathrm{Cu}_{20} \mathrm{Fe}_{10} \mathrm{Mn}_{5}$ were prepared by melting highly pure $\mathrm{Al}(99.99 \%), \mathrm{Cu}(99.99 \%), \mathrm{Fe}(99.99 \%)$, and $\mathrm{Mn}(99.99 \%)$ in an Ar atmosphere using an arc furnace.

These ingots were sealed in a quartz tube. After annealing at $800^{\circ} \mathrm{C}$ for $72 \mathrm{~h}$ and quenching in water, all the ingots produced were divided into two specimens. One specimen was used without further processing, while the other was re-annealed at $880^{\circ} \mathrm{C}$ for $72 \mathrm{~h}$ and again quenched in water. The specimens were crushed and ground into fine powder using an agate mortar. X-ray diffraction experiments were performed using $\mathrm{CuK} \alpha$ radiation with a powder diffractometer PANalytical X'pert Pro. The $\mathrm{CuK} \beta$ radiation was excluded by a Ni-filter. Rapid measurements were achieved with a silicon semiconductor array detector. Since $\mathrm{Fe}$ and $\mathrm{Mn}$ atoms produce a strong X-ray fluorescence background in diffraction patterns, weak Bragg reflections were absorbed into the background when applying the usual scan-speed. In order to measure the peak shifts precisely, the scan-speed was therefore strongly reduced over a limited angular range, to display the distinct weak peaks above the background. The position resolution of this detector corresponds to the width of the individual semiconductor devices. The reciprocal-space momentum resolution is $0.0065 \mathrm{~nm}^{-1}$, estimated from the peak profile of Si powder diffraction.

Within a quasiperiodic plane, a reciplocal-lattice vector $Q$ is specified using a five-dimensional (5D) indexing scheme ${ }^{1)}$ :

$$
\begin{array}{ll}
\boldsymbol{e}_{i}^{\|}=\sqrt{\frac{2}{5}}\left(\cos \frac{2 \pi}{5} i, \sin \frac{2 \pi}{5} i\right) & \boldsymbol{Q}_{\|}=\frac{2 \pi}{A_{5 D}} \sum_{i} n_{i} \boldsymbol{e}_{i}^{\|} \\
\boldsymbol{e}_{i}^{\perp}=\sqrt{\frac{2}{5}}\left(\cos \frac{4 \pi}{5} i, \sin \frac{4 \pi}{5} i\right) & \boldsymbol{Q}_{\perp}=\frac{2 \pi}{A_{5 D}} \sum_{i} n_{i} \boldsymbol{e}_{i}^{\perp}
\end{array}
$$

where $i=0,1,2,3$, or $4, Q_{\|}$denotes the measured momentum transfer vector, and $Q_{\perp}$ the phason momentum vector. We will henceforth use a five-dimensional (5D) indexing scheme to specify the peak indices along two orthogonal two-fold symmetry axes in the quasiperiodic plane as $m 0 \bar{n} \bar{n} 0$ and $0 m n \bar{n} \bar{m}$. By introducing an appropriate linear phason strain, the Bragg peaks move from positions corresponding to a decagonal symmetry to those of a periodic symmetry. The peak shift produced by the linear phason strain is given by

$$
\Delta Q_{\|}=M \cdot Q_{\perp}
$$

where $M$ is the phason matrix 


$$
M=\left(\begin{array}{cc}
\theta_{1} & 0 \\
0 & \theta_{2}
\end{array}\right) .
$$

Thus, in powder X-ray diffraction, the ten equivalent reflections represented by $m 0 \bar{n} \bar{n} 0$ in a decagonal symmetry separate into one kind of two equivalent reflections represented by $m 0 \bar{n} \bar{n} 0$, and two kinds of four equivalent reflections represented by $n 0 \bar{m} 0 n$ and $0 m 0 \bar{n} n$ with rectangular symmetry. Moreover, the ten equivalent reflections represented by $0 m n \bar{n} \bar{m}$ under a decagonal symmetry separate into one kind of two equivalent reflections represented by 0mnn̄ $\bar{m}$ and two kinds of four equivalent reflections represented by $n m 0 \bar{m} \bar{n}$ and $m n \bar{n} \bar{m} 0$ with rectangular symmetry.

For example, if a linear-phason strain $\theta_{1}$ is introduced, the $10 \overline{1} \overline{1} 0$ reflection has periodic indices $h=3,5,8$, and 13 for $\theta_{1}=-\tau^{-4}, \tau^{-6},-\tau^{-8}$, and $\tau^{-10}$, respectively. On the other hand, if a linear-phason strain $\theta_{2}$ is introduced, the $011 \overline{1} 1$ reflection has periodic indices $k=3,5$, and 8 for $\theta_{2}=\tau^{-5},-\tau^{-7}$, and $\tau^{-9}$, respectively. One-dimensional periodic distances are thus calculated from the shifts in the peak positions.

Moreover, by adding the index $l$ along the ten-fold symmetry periodic-axis, the above indices become $m 0 \bar{n} \bar{n} 0 l$ and $0 m n \bar{n} \bar{m} l$, respectively. In this paper, we calculate the reciprocal-space momenta $Q_{\|}$and $Q_{\perp}$ corresponding to $Q_{\|}=1 / d=$ $2 \sin \theta / \lambda$ (without the factor $2 \pi$ ).

\section{Results}

Precise measurements of the Bragg peak profiles and positions were done using powder $\mathrm{X}$-ray diffraction. Figure 1 shows the resulting patterns for $\mathrm{Al}_{65} \mathrm{Cu}_{20} \mathrm{Fe}_{7} \mathrm{Mn}_{8}$ annealed at $800^{\circ} \mathrm{C}$. Each Bragg reflection is indexed in the manner described above. All the observed reflections are consistent with the strong electron-diffraction spots in the one-dimensional quasicrystal discovered by Tasi et al. ${ }^{5)}$

The peak profiles of individual decagonal reflections are asymmetrical or are split asymmetrically. The direction of the asymmetry reflects the sign of $Q_{\perp}$, while the size of the shifts is proportional to $Q_{\perp}$. This effect is indicative of linear phason strain. To analyze this requires an accurate measurement of the reflections with a large $Q_{\perp}$. Long-duration X-ray measurements were therefore done carefully between 2.5 and $3.2 \mathrm{~nm}^{-1}$ to cover a range of $Q_{\|}$. Four peaks, indexed as $10 \overline{1} \overline{1} 00,10 \overline{1} \overline{1} 01,00 \overline{1} \overline{1} 03,10 \overline{1} \overline{1} 02$, were observed in separate

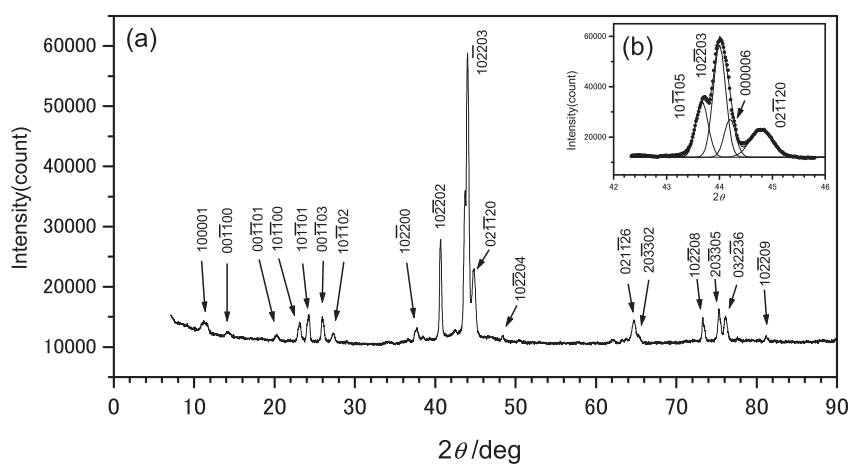

Fig. 1 X-ray diffraction patterns for $\mathrm{Al}_{65} \mathrm{Cu}_{20} \mathrm{Fe}_{7} \mathrm{Mn}_{8}$ annealed at $800^{\circ} \mathrm{C}$ (a) Clear peaks are designated $m 0 \bar{n} \bar{n} 0 l$ or $0 m n \bar{n} m l$. (b) The most intense peak overlaps several peaks. samples of different compositions (respectively, $\mathrm{Al}_{65} \mathrm{Cu}_{24} \mathrm{Fe}_{3} \mathrm{Mn}_{8}, \quad \mathrm{Al}_{65} \mathrm{Cu}_{18} \mathrm{Fe}_{7} \mathrm{Mn}_{10}, \mathrm{Al}_{65} \mathrm{Cu}_{18} \mathrm{Fe}_{9} \mathrm{Mn}_{8}$, and $\mathrm{Al}_{65} \mathrm{Cu}_{20} \mathrm{Fe}_{7} \mathrm{Mn}_{8}$ ) annealed at $800^{\circ} \mathrm{C}$ and $880^{\circ} \mathrm{C}$. Shifted peak positions were calculated and displayed together with the measured X-ray diffraction patterns (Figs. 2-4), and listed in Table 1.

Figure 2(a) shows the powder X-ray diffraction patterns for $\mathrm{Al}_{65} \mathrm{Cu}_{24} \mathrm{Fe}_{3} \mathrm{Mn}_{8}$ annealed at $800^{\circ} \mathrm{C}$. In these patterns, the peaks correspond to a decagonal type and an FCC type with $a=0.590 \mathrm{~nm}$ indicated by the arrows. For peaks of the decagonal type, the peak positions calculated using $\theta_{1}=\tau^{-6}, \theta_{2}=$ $\tau^{-5}, A_{5 D}=0.6361 \mathrm{~nm}$, and $c=1.234 \mathrm{~nm}$ most accurately reproduce the peak shifts and splittings. Here, $\theta_{1}=\tau^{-6}$ and $A_{5 D}=0.6361 \mathrm{~nm}$ result in moving the $10 \overline{1} \overline{1} 00$ peak to the periodic position with index 5 and reciprocal-space momentum $2.6242 \mathrm{~nm}^{-1}$. Similarly, $\theta_{2}=\tau^{-5}$ and $A_{5 D}=0.6361 \mathrm{~nm}$ move the $011 \overline{1} \overline{1} 0$ peak to the periodic position with index 3 and reciprocal-space momentum $2.9949 \mathrm{~nm}^{-1}$. These linear phason strains $\theta_{1}$ and $\theta_{2}$ therefore correspond to the periodicities 1.905 and $1.002 \mathrm{~nm}$ in the one-dimensional problem, respectively. The peaks indicated by arrows represent an NaCl-type structure because the clearly observed peaks obey the rule $h+$ $k+l=2 n$. On the other hand, one half of an $\mathrm{Al}_{65} \mathrm{Cu}_{24} \mathrm{Fe}_{3} \mathrm{Mn}_{8}$ ingot was melted during re-annealing at $880^{\circ} \mathrm{C}$. Figures 2(b) and 2(c) show the X-ray diffraction patterns for $\mathrm{Al}_{65} \mathrm{Cu}_{18} \mathrm{Fe}_{7} \mathrm{Mn}_{10}$ annealed at $800^{\circ} \mathrm{C}$ and re-annealed at $880^{\circ} \mathrm{C}$, respectively. The former result shows the same linear phason strain as was mentioned above. On the other hand, the latter result shows a new approximant phase of decagonal quasicrystal and a second crystalline phase. In Fig. 2(c), the splitting of the asymmetrical double peaks is clearly narrower than in Fig. 2(b). The shifts and splittings of the decagonal-type peaks in Fig. 2(c) are best reproduced with $\theta_{1}=\tau^{-10}$, $\theta_{2}=\tau^{-5}, A_{5} D=0.6368 \mathrm{~nm}$, and $c=1.234 \mathrm{~nm}$. Here, $\theta_{1}=\tau^{-10}$ makes the $10 \overline{1} \overline{1} 00$ reflection move to the periodic position with index 13 and reciprocal-space momentum $2.6033 \mathrm{~nm}^{-1}$, while $\theta_{2}=\tau^{-5}$ makes the $011 \overline{1} \overline{1} 0$ reflection move to the periodic position with index 3 and reciprocal-space momentum $2.9916 \mathrm{~nm}^{-1}$. The linear phason strains $\theta_{1}$ and $\theta_{2}$ correspond to the one-dimensional periodicities $4.994 \mathrm{~nm}$ and $1.003 \mathrm{~nm}$, respectively.

Figures 3(a) and 3(b) show the powder X-ray diffraction patterns for $\mathrm{Al}_{65} \mathrm{Cu}_{18} \mathrm{Fe}_{9} \mathrm{Mn}_{8}$ annealed at $800^{\circ} \mathrm{C}$ and re-annealed at $880^{\circ} \mathrm{C}$. In Fig. 3(a), $\theta_{1}=\tau^{-6}, \theta_{2}=\tau^{-5}, A_{5} D=$ $0.6361 \mathrm{~nm}$, and $c=1.230 \mathrm{~nm}$ yield the most accurate the peak shift. On the other hand, Fig. 3(b) shows a one-dimensional quasi-crystalline phase and another crystalline phase. Each decagonal-type peak is very narrow compared to those in Fig. 3(a). The shifts and splittings of the decagonal-type peaks in Fig. 3(b) are best reproduced with $\theta_{1}=-\tau^{-8}, \theta_{2}=0$, $A_{5 D}=0.6340 \mathrm{~nm}$, and $c=1.2285 \mathrm{~nm}$. Here, $\theta_{1}=-\tau^{-8}$ results in the $10 \overline{1} \overline{1} 00$ reflection moving to the periodic position with index 8 and reciprocal-space momentum $2.6035 \mathrm{~nm}^{-1}$. This linear phason strain $\theta_{1}$ corresponds to the one-dimensional periodicicity $3.0728 \mathrm{~nm}$. The phase transformation from the approximant to the one-dimensional quasicrystal is therefore produced by re-annealing at $880^{\circ} \mathrm{C}$.

Figures 4(a) and 4(b) show the powder X-ray diffraction patterns for $\mathrm{Al}_{65} \mathrm{Cu}_{20} \mathrm{Fe}_{7} \mathrm{Mn}_{8}$ annealed at $800^{\circ} \mathrm{C}$ and re-annealed at $880^{\circ} \mathrm{C}$. In Fig. $4(\mathrm{a})$, setting $\theta_{1}=-\tau^{-6}, \theta_{2}=0, A_{5 D}=$ 
Table 1 Calculation of the ideal peak position and the split-peak positions for decagonal quasicrystals.

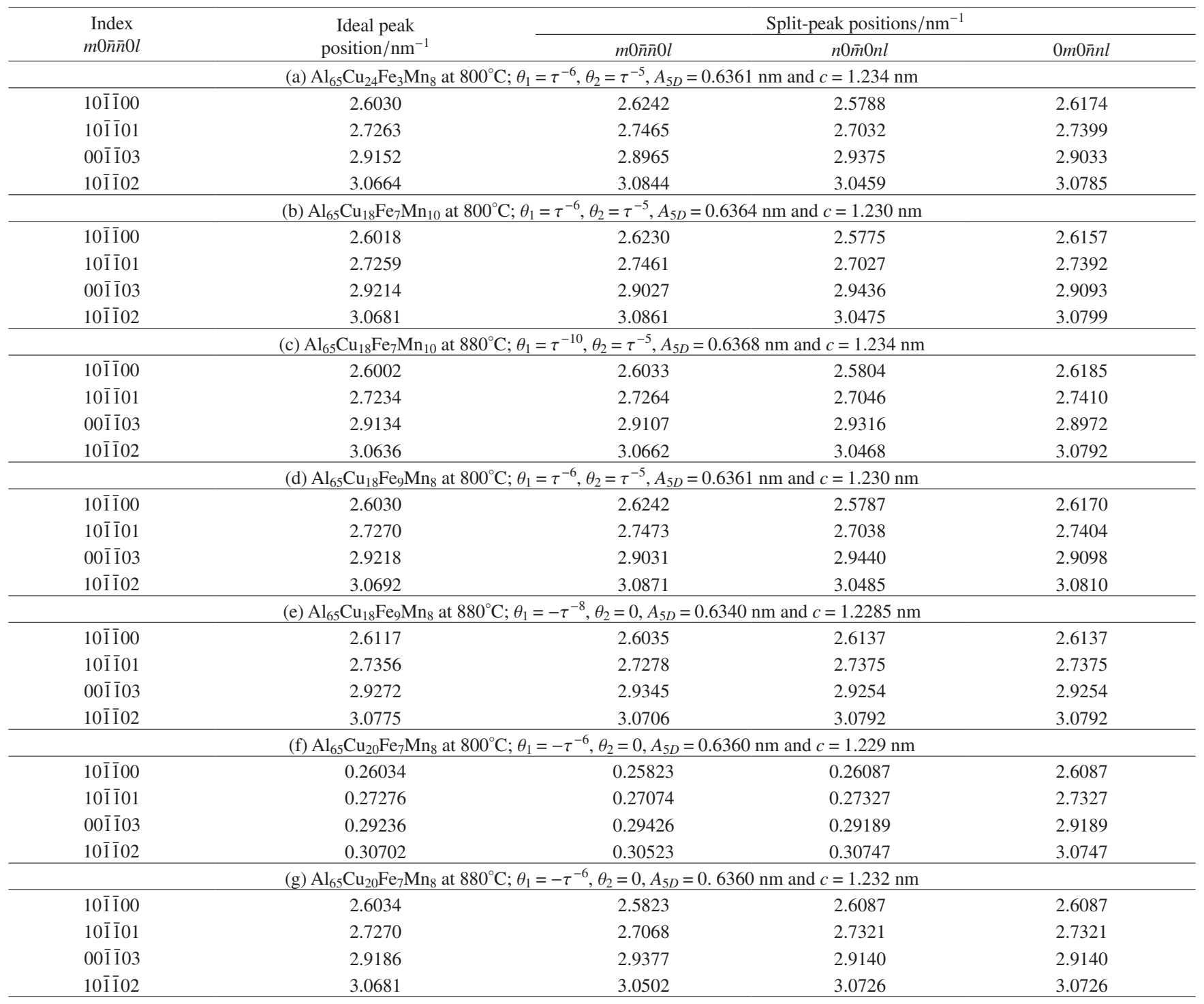

$0.6360 \mathrm{~nm}$, and $c=1.229 \mathrm{~nm}$ reproduces the peak shift most accurately. Here, $\theta_{1}=-\tau^{-6}$ makes the $10 \overline{1} \overline{1} 00$ reflection move to the periodic position with index 11 and reciprocal momentum $0.25823 \mathrm{~nm}^{-1}$. This linear phason strain $\theta_{1}$ corresponds to the one-dimensional periodicity $4.2598 \mathrm{~nm}$. This phase is one of the one-dimensional quasicrystals discovered by Yang et al. ${ }^{6)}$ and is stable at $800^{\circ} \mathrm{C}$ and at $880^{\circ} \mathrm{C}$.

In Table1, from the comparison between compositions and lattice constants $A_{5 \mathrm{D}}$ and $c$, excluding (e) one-dimensional quasicrystal of $\theta_{1}=-\tau^{-8}, \theta_{2}=0$, as Fe increases and $\mathrm{Cu}$ decreases along (a)-(d)-(f), and Mn decreases and $\mathrm{Cu}$ increases along (b)-(f) and (c)-(g), the lattice constant tends to decrease somewhat.

\section{Discussion}

We found two types of approximant for the AlCuFeMn decagonal quasicrystal, with linear phason strains $\theta_{1}=\tau^{-6}$ and $\theta_{2}=\tau^{-5}$, and $\theta_{1}=\tau^{-10}$ and $\theta_{2}=\tau^{-5}$, respectively. The first type of approximant is formed from the one-dimensional quasicrystal with $\theta_{1}=\tau^{-6}$ and $\theta_{2}=0$, found by Tsai et al. ${ }^{5)}$, by intro- ducing the further linear phason strain $\theta_{2}=\tau^{-5}$. The second type of approximant is formed from the first type of approximant by a $\tau^{4}$-fold decrease in $\theta_{1}$, corresponds to a $\tau^{2}$ - fold increase in the periodicity.

We simulated the peak shifts due to linear phason strains on the quasi-periodic plane, and calculated the lattice constants of the approximants. Figure 5 (a) shows the peak positions for an ideal decagonal quasicrystal. The peak positions of the $10 \overline{1} \overline{1} 0$ and $011 \overline{1} \overline{1}$ reflections are indicated by arrows on the $Q_{\| x}(m 0 \bar{n} \bar{n} 0)$ and $Q_{\| y}(0 m n \bar{n} \bar{m})$ axes in the quasi-periodic plane, respectively.

Figure 5 (b) shows the shifts in the peak positions induced by the linear phason strains $\theta_{1}=\tau^{-6}$ and $\theta_{2}=\tau^{-5}$, as determined using eqs. (2) and (3). As a result of these shifts, the peak positions refer to an approximant with an orthorhombic reciprocal lattice. The shifted $10 \overline{1} \overline{1} 0$ reflection is located in the tenth cell from the center and corresponds to a reciprocal-space momentum of $2.6242 \mathrm{~nm}^{-1}$, as calculated using $A_{5 D}=0.6361 \mathrm{~nm}$ and $\theta_{1}=\tau^{-6}$. The estimated lattice constant $a=3.811 \mathrm{~nm}$ is hence double that of the one-dimensional periodicity $1.905 \mathrm{~nm}$. The odd-index peaks on the $Q_{\| \mathrm{x}}$-axis fall 

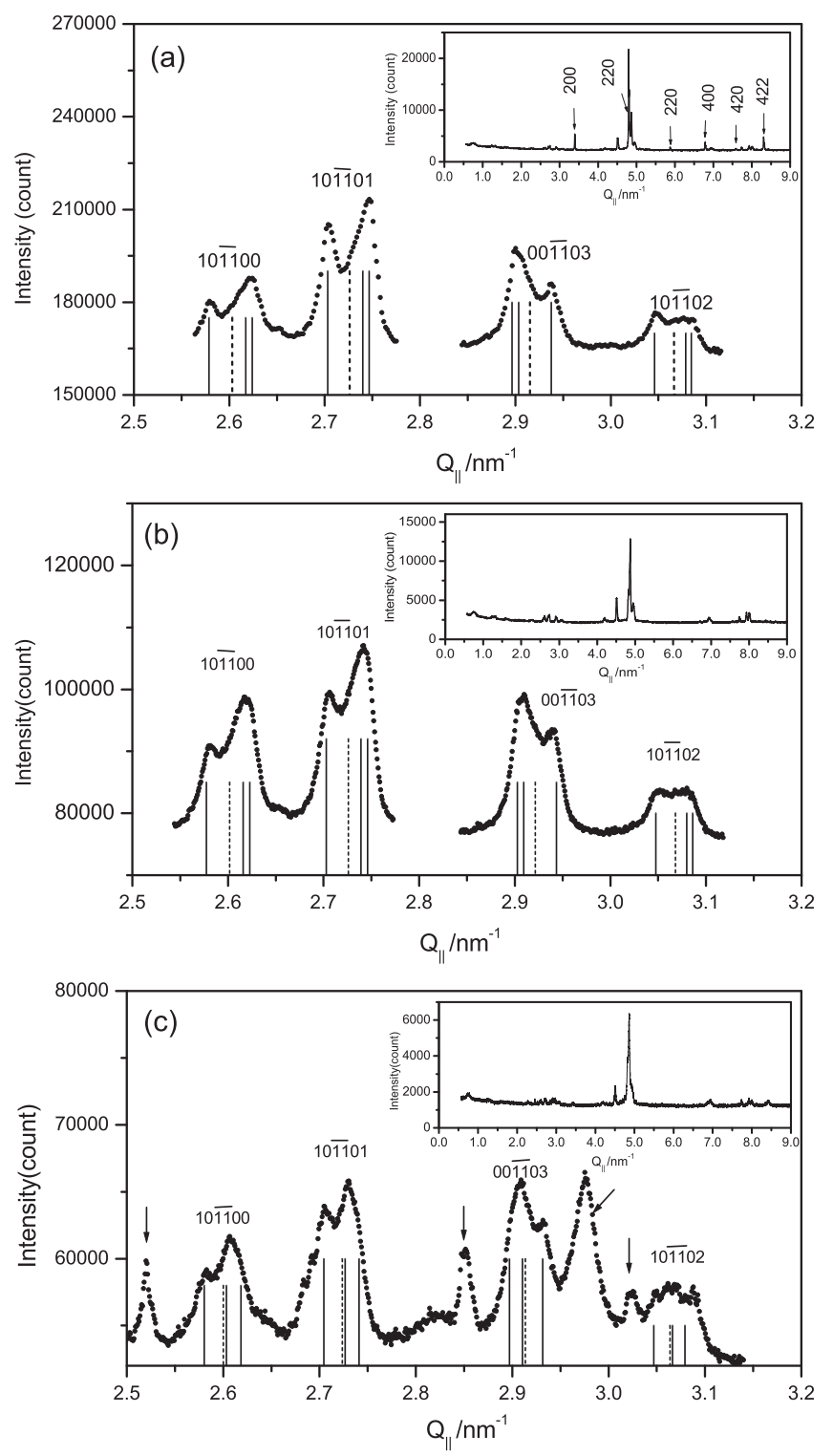

Fig. 2 Peak profiles and simulated peak positions for (a) $\mathrm{Al}_{65} \mathrm{Cu}_{24} \mathrm{Fe}_{3} \mathrm{Mn}_{8}$ annealed at $800^{\circ} \mathrm{C}$, and $\mathrm{Al}_{65} \mathrm{Cu}_{18} \mathrm{Fe}_{7} \mathrm{Mn}_{10}$ annealed at (b) $800^{\circ} \mathrm{C}$ and (c) $880^{\circ} \mathrm{C}$. The dotted lines represent identical decagonal peak positions, and the solid lines the split-peak positions shifted relative to the identical positions as a result of the linear phason strains (a) $\theta_{1}=\tau^{-6}$ and $\theta_{2}=\tau^{-5}$, and (b) $\theta_{1}=\tau^{-10}$ and $\theta_{2}=\tau^{-5}$. The peaks indicated by arrows in (a) describe an $\mathrm{NaCl}$-type structure. Moreover, the peaks indicated by arrows in (c) indicate crystalline phases.

outside of this axis upon application of the one-dimensional periodicity. Similarly, the shifted $011 \overline{1} \overline{1}$ reflection is located in the sixth cell from the center and has $a$ reciprocal-space momentum of $1.9966 \mathrm{~nm}^{-1}$, as calculated using $A_{5 D}=$ $0.6361 \mathrm{~nm}$ and $\theta_{2}=\tau^{-5}$. The estimated lattice constant $b=$ $2.003 \mathrm{~nm}$ is therefore double the one-dimensional periodicity $1.002 \mathrm{~nm}$. The odd-index peaks on the $Q_{\| \mathrm{y}}$-axis enter this window from outside of the window range upon application of the one-dimensional periodicity. Figure 5 (c) shows the orthorhombic peak positions shifted by the linear phason strains $\theta_{1}=\tau^{-10}$ and $\theta_{2}=\tau^{-5}$. The shifted $10 \overline{1} \overline{1} 0$ and $011 \overline{1} \overline{1}$ reflections are located in the twenty-sixth and the sixth cells from the center and have reciprocal-space momentums of $2.6033 \mathrm{~nm}^{-1}$ and $2.9916 \mathrm{~nm}^{-1}$, calculated using $A_{5 D}=0.6368 \mathrm{~nm}$, and $\theta_{1}=$
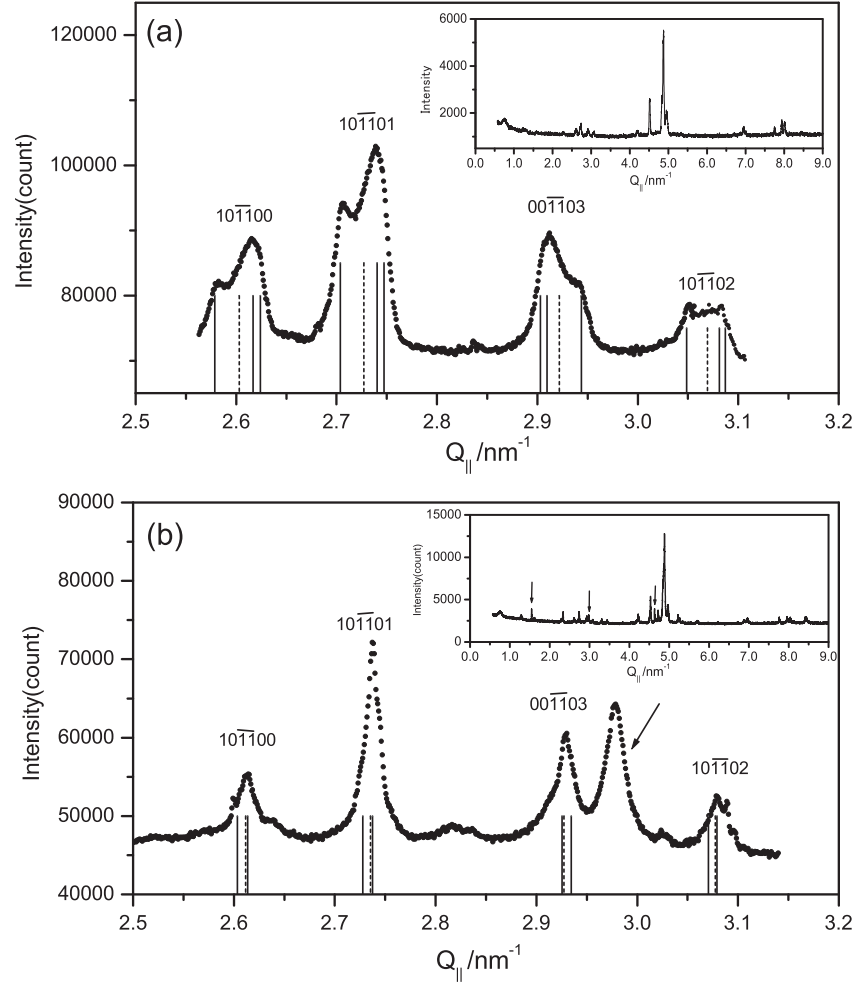

Fig. 3 Peak profiles and simulated peak positions for $\mathrm{Al}_{65} \mathrm{Cu}_{18} \mathrm{Fe}_{9} \mathrm{Mn}_{8}$ (a) annealed at $800^{\circ} \mathrm{C}$ and (b) re-annealed at $880^{\circ} \mathrm{C}$, respectively. The dotted lines represent identical decagonal peak positions and solid lines splitpeak positions shifted relative to the identical positions as a result of the linear phason strains (a) $\theta_{1}=\tau^{-6}$ and $\theta_{2}=\tau^{-5}$, and (b) $\theta_{1}=-\tau^{-8}$ and $\theta_{2}=$ 0 . The peaks indicated by arrows in (b) show some crystalline phases.
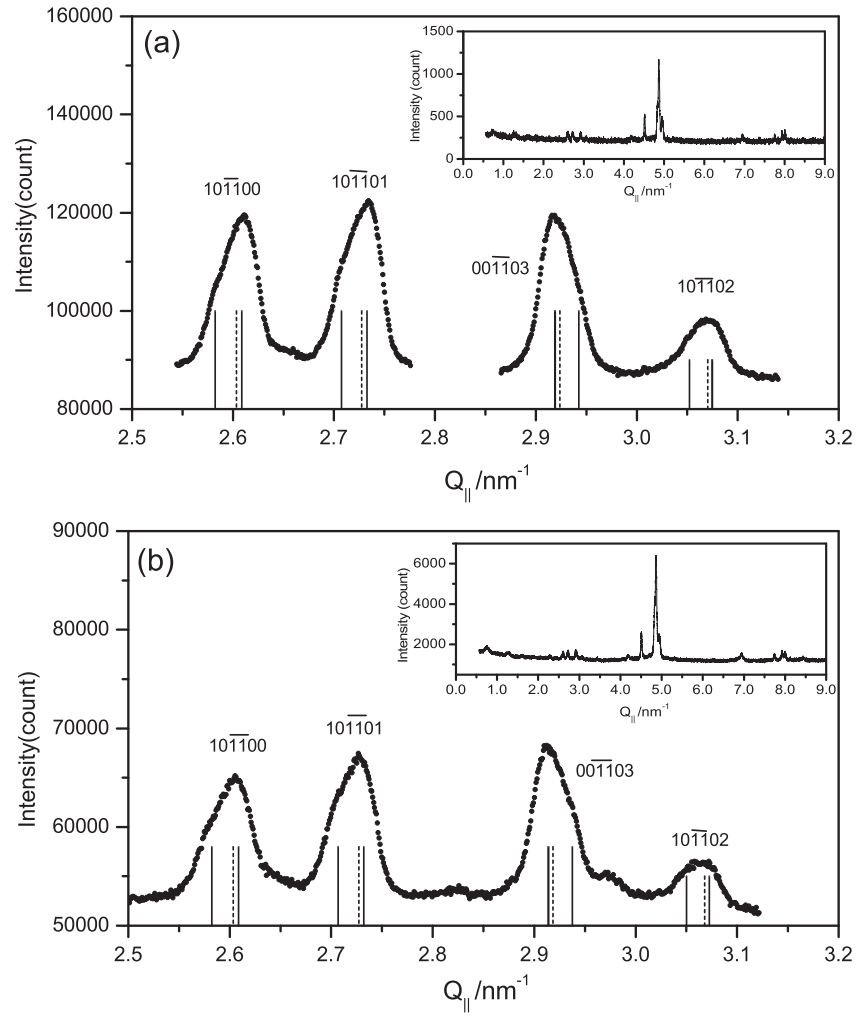

Fig. 4 Peak profiles and simulated peak positions for $\mathrm{Al}_{65} \mathrm{Cu}_{20} \mathrm{Fe}_{7} \mathrm{Mn}_{8}$ (a) annealed at $800^{\circ} \mathrm{C}$ and (b) re-annealed at $880^{\circ} \mathrm{C}$, respectively. The dotted lines represent identical decagonal peak positions and the solid lines the split-peak positions shifted relative to the identical positions as a result of the linear phason strains $\theta_{1}=-\tau^{-6}, \theta_{2}=0$. 

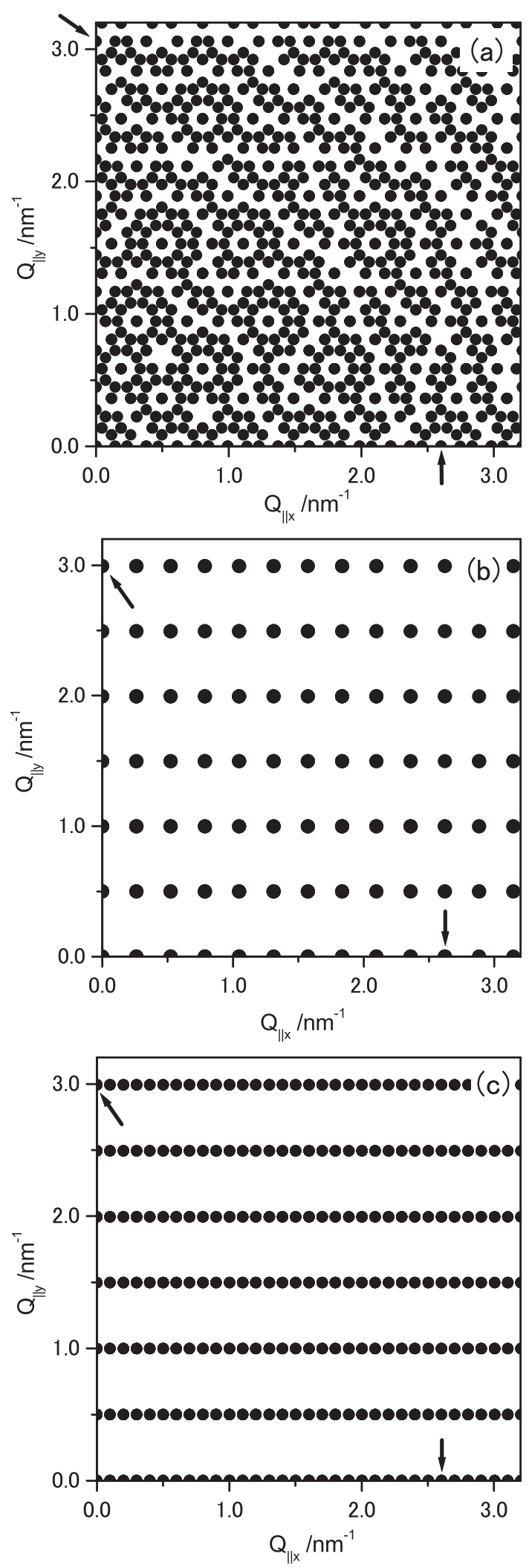

Fig. 5 Simulated peak positions in a quasi-periodic plane for: (a) an ideal decagonal quasicrystal; (b) an approximant crystal with linear phason strains of $\theta_{1}=\tau^{-6}$ and $\theta_{2}=\tau^{-5}$, with lattice constants $a=3.811 \mathrm{~nm}$ and $\mathrm{b}=$ $2.003 \mathrm{~nm}$; (c) an approximant crystal with linear phason strains $\theta_{1}=\tau^{-10}$ and $\theta_{2}=\tau^{-5}$, with lattice constants $a=9.999 \mathrm{~nm}$ and $b=2.003 \mathrm{~nm}$. Peak positions at the $10 \overline{1} \overline{1} 0$ and $011 \overline{1} \overline{1}$ reflections are denoted by arrows on the $Q_{\| x}(m 0 \bar{n} \bar{n} 0)$ and $Q_{\| y}(0 m n \bar{n} \bar{m})$ axes, respectively. $\tau^{-10}$ and $\theta_{1}=\tau^{-5}$, respectively. Therefore, the estimated lattice constant $\mathrm{a}=9.998 \mathrm{~nm}$ is double the one-dimensional periodicity $4.994 \mathrm{~nm}$. Similarly, the lattice constant $b=$ $2.006 \mathrm{~nm}$ is calculated to be double the one-dimensional periodicity $1.003 \mathrm{~nm}$.

The first type of approximant appears at $800^{\circ} \mathrm{C}$ with compositions $\quad \mathrm{Al}_{65} \mathrm{Cu}_{18} \mathrm{Fe}_{9} \mathrm{Mn}_{8}, \quad \mathrm{Al}_{65} \mathrm{Cu}_{18} \mathrm{Fe}_{7} \mathrm{Mn}_{10}, \quad$ and $\mathrm{Al}_{65} \mathrm{Cu}_{24} \mathrm{Fe}_{3} \mathrm{Mn}_{8}$. The second type of approximant appears at $880^{\circ} \mathrm{C}$, with a composition $\mathrm{Al}_{65} \mathrm{Cu}_{18} \mathrm{Fe}_{7} \mathrm{Mn}_{10}$, and transforms into the first type. Moreover, we confirmed the existence of two-types of one-dimensional quasicrystal, which have linear phason strains $\theta_{1}=\tau^{-6}$ and $\theta_{2}=0$, and $\theta_{1}=-\tau^{-8}$ and $\theta_{2}=0$, found by Yang et al $^{6)}$ For the composition of $\mathrm{Al}_{65} \mathrm{Cu}_{20} \mathrm{Fe}_{7} \mathrm{Mn}_{8}$, the first type of approximant, with $\theta_{1}=\tau^{-6}$ and $\theta_{2}=\tau^{-5}$ at $800^{\circ} \mathrm{C}$, changes into a one-dimensional quasicrystal with $\theta_{1}=$ $-\tau^{-8}$ and $\theta_{2}=0$ at $880^{\circ} \mathrm{C}$. Furthermore, for the composition of $\mathrm{Al}_{65} \mathrm{Cu}_{20} \mathrm{Fe}_{10} \mathrm{Mn}_{5}$, located near the composition of $\mathrm{Al}_{65} \mathrm{Cu}_{20} \mathrm{Fe}_{7} \mathrm{Mn}_{8}$, studied by Tasi et al. ${ }^{5)}$ and Yang et al. ${ }^{6}$, there are three-types of one-dimensional quasicrystals with $\theta_{1}=\tau^{-6}$ and $\theta_{2}=0, \theta_{1}=-\tau^{-8}$ and $\theta_{2}=0$, and $\theta_{1}=-\tau^{-6}$ and $\theta_{2}=0$ at $880^{\circ} \mathrm{C}$. Therefore, an ideal decagonal quasicrystyal may be stable above $880^{\circ} \mathrm{C}$ around these compositions.

On the other hand, a one-dimensional quasicrystal with $\theta_{1}=-\tau^{-6}$ and $\theta_{2}=0$ is formed at $800^{\circ} \mathrm{C}$ and $880^{\circ} \mathrm{C}$ with composition $\mathrm{Al}_{65} \mathrm{Cu}_{20} \mathrm{Fe}_{7} \mathrm{Mn}_{8}$. This linear phason strain $\theta_{1}=-\tau^{-6}$ is another type compared to the series of the above-mentioned linear phason strains $\theta_{1}=\tau^{-6},-\tau^{-8}$, and $\tau^{-10}$.

The AlCuFeMn alloy system can consist of a one-dimensional quasicrystal and many approximants, each with a different linear phason strain. In the future, an ideal decagonal crystal may be found at a higher temperature. Further experiments on $\mathrm{AlCuFeMn}$ alloy systems will yield more detailed information on the transformation from a decagonal quasicrystal to approximants via one-dimensional quasicrystals.

\section{Conclusion}

We discovered two types of approximants in an AlCuFeMn alloy system, arising from the linear phason strains $\theta_{1}=\tau^{-6}$ and $\theta_{2}=\tau^{-5}$, and $\theta_{1}=\tau^{-10}$ and $\theta_{2}=\tau^{-5}$. These correspond, respectively, to $a=3.811 \mathrm{~nm}$ and $b=2.003 \mathrm{~nm}$, and $a=$ $9.998 \mathrm{~nm}$ and $b=2.006 \mathrm{~nm}$ for an orthorhombic system.

\section{REFERENCES}

1) H. Zhang and K.H. Kuo: Phys. Rev. B 41 (1990) 3482-3487

2) Y. Matsuo, Y. Nishimura, K. Yamamoto and Y. Ishii: Mater. Trans. 42 (2001) 2008-2012.

3) K. Yamamoto, W. Yang, Y. Nishimura, N. Ikeda and Y. Masuo: Mater. Trans. 45 (2004) 1255-1260.

4) Y. Matsuo, K. Yamamoto and Y. Ishii: J. Phys. Condens. Matter 10 (1998) 983-994.

5) A.P. Tsai, A. Sato, A. Yamamoto, A. Inoue and T. Masumoto: Jpn. J. Appl. Phys. 31 (1992) L970-L973.

6) W.G. Yang, J.N. Gui and R.H. Wang: Philos. Mag. Lett. 74 (1996) 357366. 